\title{
Convergence analysis of projection methods for a new system of general nonconvex variational inequalities
}

\author{
Dao-Jun Wen ${ }^{*}$, Xian-Jun Long ${ }^{1}$ and Qian-Fen Gong ${ }^{2}$
}

\author{
* Correspondence: \\ daojunwen@163.com \\ ${ }^{1}$ College of Mathematics and \\ Statistics, Chongqing Technology \\ and Business University, \\ Chongqing 400067, China \\ Full list of author information is \\ available at the end of the article
}

\begin{abstract}
In this article, we introduce and consider a new system of general nonconvex variational inequalities defined on uniformly prox-regular sets. We establish the equivalence between the new system of general nonconvex variational inequalities and the fixed point problems to analyze an explicit projection method for solving this system. We also consider the convergence of the projection method under some suitable conditions. Results presented in this article improve and extend the previously known results for the variational inequalities and related optimization problems.

MSC (2000): 47J20; 47N10; 49J30.

Keywords: system of general nonconvex variational inequalities, explicit projection methods, uniform prox-regular set, $r$-strongly monotone mappings, $\mu$-Lipschitz continuous.
\end{abstract}

\section{Introduction}

Variational inequalities theory, which was introduced by Stampacchia [1], has emerged as an interesting and fascinating branch of mathematical and engineering sciences. The ideas and techniques of variational inequalities are being applied in structural analysis, economics, optimization, operations research fields. It has been shown that variational inequalities provide the most natural, direct, simple, and efficient framework for a general treatment of some unrelated problems arising in various fields of pure and applied sciences. In recent years, there have been considerable activities in the development of numerical techniques including projection methods, Wiener-Hopf equations, auxiliary principle, and descent framework for solving variational inequalities; see [2-17] and the references therein. These activities have motivated us to generalize and extend the variational inequalities and related optimization problems in several directions using novel techniques.

Projection technique has played a significant role in the numerical solution of variational inequalities based on the convergence analysis. It is worth mentioning that almost all the results regarding the existence and iterative schemes for variational inequalities, which have been investigated and considered, if the underlying set is a convex set. This is because all the techniques are based on the properties of the projection operator over convex sets, which may not hold in general, when the sets are

(C) 2012 Wen et al; licensee Springer. This is an Open Access article distributed under the terms of the Creative Commons Attribution License (http://creativecommons.org/licenses/by/2.0), which permits unrestricted use, distribution, and reproduction in any medium, provided the original work is properly cited. 
nonconvex. Recently, Clarke et al. [8] and Poliquin et al. [9] have introduced and studied a new class of nonconvex sets, which are called uniformly prox-regular sets. It is known that uniformly prox-regular sets are nonconvex sets and include convex sets as a special case. This class of uniformly prox-regular sets has played an important part in many nonconvex applications such as optimization, dynamical systems, and differential inclusions.

In 2009, Noor [10] introduced a nonconvex variational inequalities based on the uniformly prox-regular sets. Moreover, he discussed the existence and algorithm of the solution for the nonconvex variational inequalities, which shows projection technique can be extended to nonconvex sets. Noor [11] proposed some iterative methods for solving a general nonconvex variational inequalities with projection methods and Wiener-Hopf equations technique. On the other hand, Verma [13] and Noor and Noor [14] proposed explicit projection methods for solving systems of variational inequalities and general variational inequalities on a closed convex subset of Hilbert space, respectively. Very recently, Wen [15] modified projection methods to a generalized system of nonconvex variational inequalities with different nonlinear operators. However, only iterative sequences $\left\{g\left(x_{n}\right)\right\},\left\{g\left(y_{n}\right)\right\}$ come from the projection methods, which requires that mapping $g$ must be injective in order to arrive at a solution of the generalized system. Furthermore, the property defined on the underlying operator $T$ depends on the mapping $g$ in convergence analysis. These strict conditions rule out many applications of the projection type methods for the generalized system of nonconvex variational inequalities.

In this article, motivated and inspired by the research going on in this direction, we introduce and consider a more general system, which is called a new general nonconvex variational inequalities. The new system includes the system of variational inequalities involving two different nonlinear operators, the general nonconvex variational inequalities and the systems of variational inequalities defined on closed convex sets as special cases.

The purpose of this article is not only to show that projection technique can be extended to the new system of general nonconvex variational inequalities on uniformly prox-regular sets, but also to get rid of the dependence of $T$ on the mapping $g$ and the injective property defined on $g$ in convergence analysis of the projection method for solving the new system of general nonconvex variational inequalities. Our results extend and improve the corresponding results of [7,10-15].

\section{Preliminaries}

Let $H$ be a real Hilbert space whose inner product and norm are denoted by $\langle. .$.$\rangle and$ $\|$.$\| , respectively. Let K$ be a nonempty and convex subset in $H$.

First of all, we recall the following well-known concepts from nonlinear convex analysis and non-smooth analysis $[8,9]$.

Definition 2.1. The proximal normal cone of $K$ at $u \in H$ is given by

$$
N_{K}^{P}(u):=\left\{\xi \in H: u \in P_{K}[u+\alpha \xi]\right\},
$$

where $\alpha>0$ is a constant and

$$
P_{K}[u]=\left\{u^{*} \in K: d_{K}(u)=\left\|u-u^{*}\right\|\right\} .
$$


Here $d_{K}($.$) is the usual distance function to the subset K$, that is $d_{K}(u)=\inf _{v \in K} \| v$ $u \|$. The proximal normal cone $N_{K}^{P}(u)$ has the following characterization.

Lemma 2.1. Let $K$ be a nonempty, closed, and convex subset in $H$. Then $\varsigma \in N_{K}^{P}(u)$ if and only if there exists a constant $\alpha=\alpha(\zeta, u)>0$ such that

$$
\langle\varsigma, v-u\rangle \leq \alpha\|v-u\|^{2}, \quad \forall v \in K .
$$

Definition 2.2. The Clarke normal cone, denoted by $N_{K}^{C}(u)$, is defined as

$$
N_{K}^{C}(u)=\overline{c o}\left[N_{K}^{P}(u)\right]
$$

where $\overline{c o}$ means the closure of the convex hull. Clearly $N_{K}^{P}(u) \subset N_{K}^{C}(u)$, but the converse is not true. Note that $N_{K}^{C}(u)$ is always closed and convex, whereas $N_{K}^{P}(u)$ is convex, but may not be closed [9].

Definition 2.3. For a given $r \in(0, \infty]$, a subset $K_{r}$ is said to be normalized uniformly $r$-prox-regular if and only if every nonzero proximal normal to $K_{r}$ can be realized by an $r$-ball, that is, $\forall u \in K_{r}$ and $0 \neq \xi \in N_{K_{r}}^{P}(u)$, one has

$$
\left\langle\frac{\xi}{\|\xi\|}, v-u\right\rangle \leq \frac{1}{2 r}\|v-u\|^{2}, \quad \forall v \in K_{r} .
$$

It is clear that the class of normalized uniformly prox-regular sets is sufficiently large to include the class of convex sets, $p$-convex sets, $C^{1,1}$ submanifolds (possibly with boundary) of $H$, the images under a $C^{1,1}$ diffeomorphism of convex sets and many other nonconvex sets; see [8,9]. It is known that if $K_{r}$ is a uniformly prox-regular set, then the proximal normal cone $N_{K_{r}}^{P}(u)$ is closed as a set-valued mapping. Thus, we have $N_{K_{r}}^{P}(u)=N_{K_{r}}^{C}(u)$.

Remark 2.1. It is clear that if $r=\infty$, then uniformly prox-regularity of $K_{r}$ is equivalent to the convexity of $K$, that is, $K_{r}=K$.

Let $K_{r}$ be a uniformly $r$-prox-regular (nonconvex) set, and $T_{1}, T_{2}: K_{r} \times K_{r} \rightarrow K_{r}$ and $g, h: H \rightarrow K_{r}$ be different nonlinear operators, respectively. For any given constants $\rho$ $>0$ and $\eta>0$, we consider the problem of finding $x^{*}, y^{*} \in K_{r}$ such that

$$
\begin{aligned}
& \left\langle\rho T_{1}\left(y^{*}, x^{*}\right)+x^{*}-g\left(\gamma^{*}\right), g(x)-x^{*}\right\rangle+\frac{1}{2 r}\left\|g(x)-x^{*}\right\|^{2} \geq 0, \quad \forall x \in H: g(x) \in K_{r}, \\
& \left\langle\eta T_{2}\left(x^{*}, y^{*}\right)+y^{*}-h\left(x^{*}\right), h(x)-y^{*}\right\rangle+\frac{1}{2 r}\left\|h(x)-y^{*}\right\|^{2} \geq 0, \quad \forall x \in H: h(x) \in K_{r},
\end{aligned}
$$

which is called a new system of general nonconvex variational inequalities.

If $g=h=I$, the identity operator, then problem (2.1) is equivalent to finding $x^{*}, y^{*} \in$ $K_{r}$ such that

$$
\begin{aligned}
& \left\langle\rho T_{1}\left(y^{*}, x^{*}\right)+x^{*}-y^{*}, x-x^{*}\right\rangle+\frac{1}{2 r}\left\|x-x^{*}\right\|^{2} \geq 0, \quad \forall x \in K_{r}, \rho>0, \\
& \left\langle\eta T_{2}\left(x^{*}, y^{*}\right)+y^{*}-x^{*}, x-y^{*}\right\rangle+\frac{1}{2 r}\left\|x-y^{*}\right\|^{2} \geq 0, \quad \forall x \in K_{r}, \eta>0
\end{aligned}
$$

which appears to be the other new system of nonconvex variational inequalities.

We note that, if $r=\infty, K_{r}=K$, the convex subset in $H$, then problem (2.1) is equivalent to finding $x^{*}, y^{*} \in K$ such that 


$$
\begin{aligned}
& \left\langle\rho T_{1}\left(y^{*}, x^{*}\right)+x^{*}-g\left(y^{*}\right), g(x)-x^{*}\right\rangle \geq 0, \quad \forall x \in H: g(x) \in K, \rho>0, \\
& \left\langle\eta T_{2}\left(x^{*}, y^{*}\right)+y^{*}-h\left(x^{*}\right), h(x)-y^{*}\right\rangle \geq 0, \quad \forall x \in H: h(x) \in K, \eta>0,
\end{aligned}
$$

which is known as the system of general variational inequalities involving four different nonlinear operators, introduced, and studied by Noor and Noor [14].

If $g=h=I, T_{1}, T_{2}: K \rightarrow K$ are two univariate nonlinear operators, then problem (2.3) is equivalent to finding $x^{*}, y^{*} \in K$ such that

$$
\begin{aligned}
& \left\langle\rho T_{1}\left(y^{*}\right)+x^{*}-y^{*}, x-x^{*}\right\rangle \geq 0, \quad \forall x \in K, \rho>0, \\
& \left\langle\eta T_{2}\left(x^{*}\right)+y^{*}-x^{*}, x-y^{*}\right\rangle \geq 0, \quad \forall x \in K, \eta>0,
\end{aligned}
$$

which is known as the system of nonlinear variational inequalities involving two different nonlinear operators. If $T_{1}=T_{2}$, problem (2.4) reduces to the system of variational inequalities, which was introduced and studied by Verma [13].

It is worth mentioning that if $T_{1}=T_{2}=T: K_{r} \rightarrow K_{r}$ is a univariate nonlinear operator, and $x^{*}=y^{*}=u$, then problem (2.2) reduces to finding $u \in K_{r}$ such that

$$
\langle T u, v-u\rangle+\frac{1}{2 r}\|v-u\|^{2} \geq 0, \quad \forall v \in K_{r},
$$

which is more general than the normal nonconvex variational inequality, introduced and studied by Bounkhel et al. [3] and Noor [7,10], that is

$$
\langle T u, v-u\rangle \geq 0, \quad \forall v \in K_{r} .
$$

It is well known that problem (2.6) is equivalent to finding $u \in K_{r}$ such that

$$
0 \in T u+N_{K_{r}}^{P}(u),
$$

where $N_{K_{r}}^{P}(u)$ denotes the normal cone of $K_{r}$ at $u$ in the sense of nonconvex analysis. Problem (2.7) is called the variational inclusion associated with nonconvex variational inequality (2.6), which implies that the nonconvex variational inequality is equivalent to finding a zero of the sum of two monotone operators. This equivalent formulation plays a crucial and basic part in this article, which allows us to use the projection operator technique for solving the general system of nonconvex variational inequalities (2.1).

We now recall the well-known properties of the uniform prox-regular sets [8-10,15].

Lemma 2.2. Let $K$ be a nonempty closed subset of $H, r \in(0, \infty]$ and set $K_{r}=\{u \in H$ $: d(u, K)<r\}$. If $K_{r}$ is uniformly prox-regular, then

(i) $\forall u \in K_{r}, P_{K_{r}}(u) \neq 0$..

(ii) $\forall r^{\prime} \in(0, r), P_{K_{r}}$ is Lipschitz continuous with constant $\delta=\frac{r}{r-r^{\prime}}$ on $K_{r^{\prime}}$.

(iii) The proximal normal cone is closed as a set-valued mapping.

Lemma 2.3 [18]. Assume $\left\{\alpha_{n}\right\}$ is a sequence of nonnegative real numbers such that

$$
\alpha_{n+1} \leq\left(1-\gamma_{n}\right) \alpha_{n}+\sigma_{n}, \quad n \geq 0,
$$

where $\left\{\gamma_{n}\right\}$ is a sequence in $(0,1)$ and $\left\{\sigma_{n}\right\}$ is a sequence in $R$ such that

(i) $\sum_{n=0}^{\infty} \gamma_{n}=\infty$;

(ii) $\lim \sup _{n \rightarrow \infty} \sigma_{n} / \gamma_{n} \leq 0$ or $\sum_{n=0}^{\infty}\left|\sigma_{n}\right|<\infty$. 
Then $\lim _{n \rightarrow \infty} a_{n}=0$.

Definition 2.4. An operator $T: H \rightarrow H$ is said to be $r$-strongly monotone, if there exists a constant $r>0$ such that

$$
\langle T x-T y, x-y\rangle \geq r\|x-y\|^{2}, \quad \forall x, y \in H .
$$

Definition 2.5. An operator $T: H \rightarrow H$ is said to be $\mu$-Lipschitz continuous, if there exists a constant $\mu>0$ such that

$$
\|T x-T y\| \leq \mu\|x-y\|, \quad \forall x, y \in H .
$$

Remark 2.2. As $T=I$, the identity operator is 1 -strongly monotone and 1-Lipschitz continuous.

Remark 2.3. Obviously, whenever operator $T$ is $r$-strongly monotone and $\mu$-Lipschitz continuous, it follows that $\mu \geq r$.

\section{Projection methods}

In this section, we establish the equivalence between the new system of general nonconvex variational inequalities (2.1) and the fixed point problem with the projection technique. This alternative formulation enable us to suggest and analyze an explicit projection method for solving system (2.1).

Lemma 3.1. $x^{*}, y^{*} \in K_{r}$ is a solution of the system of general nonconvex variational inequalities (2.1), if and only if

$$
\begin{aligned}
& x^{*}=P_{K_{r}}\left[g\left(y^{*}\right)-\rho T_{1}\left(y^{*}, x^{*}\right)\right], \\
& y^{*}=P_{K_{r}}\left[h\left(x^{*}\right)-\eta T_{2}\left(x^{*}, y^{*}\right)\right],
\end{aligned}
$$

where $P_{K_{r}}$ is the projection of $H$ onto the uniformly prox-regular set $K_{r}$.

Proof. Let $x^{*}, y^{*} \in K_{r}$ be a solution of (2.1). From (2.7), we have that the problem (2.1a) is equivalent to that

$$
0 \in \rho T_{1}\left(\gamma^{*}, x^{*}\right)+x^{*}-g\left(\gamma^{*}\right)+N_{K_{r}}^{P}\left(x^{*}\right) .
$$

where $N_{K_{r}}^{P}\left(x^{*}\right)$ is proximal normal cone of $K_{r}$ at $x^{*}$ in the sense of nonconvex analysis. Indeed, if $\rho T_{1}\left(y^{*}, x^{*}\right)+x^{*}-g\left(y^{*}\right)=0$, because the vector zero always belongs to any normal cone, then (3.2) is valid. If $\rho T_{1}\left(y^{*}, x^{*}\right)+x^{*}-g\left(y^{*}\right) \neq 0$, then for all $x \in H$ : $g(x) \in K_{r}$, it follows from (2.1a) that

$$
\left\langle-\left(\rho T_{1}\left(\gamma^{*}, x^{*}\right)+x^{*}-g\left(\gamma^{*}\right)\right), g(x)-x^{*}\right\rangle \leq \frac{1}{2 r}\left\|g(x)-x^{*}\right\|^{2} .
$$

By using Lemma 2.1, we obtain

$$
-\left(\rho T_{1}\left(\gamma^{*}, x^{*}\right)+x^{*}-g\left(y^{*}\right)\right) \in N_{K_{r}}^{P}\left(x^{*}\right)
$$

and so (3.2) holds also. Consequently, the general nonconvex variational inequality (2.1a) is equivalent to (3.2), which is called variational inclusion associated with the problem (2.1a).

On the other hand, (3.2) can be written as

$$
g\left(y^{*}\right)-\rho T_{1}\left(\gamma^{*}, x^{*}\right) \in x^{*}+N_{K_{r}}^{P}\left(x^{*}\right)=\left(I+N_{K_{r}}^{P}\right) x^{*},
$$


where $I$ is identity operator. Moreover, we have

$$
x^{*}=P_{K_{r}}\left[g\left(y^{*}\right)-\rho T_{1}\left(\gamma^{*}, x^{*}\right)\right],
$$

where we have used the well-known fact that $P_{K_{r}}=\left(I+\rho N_{K_{r}}^{P}\right)^{-1}$. In a similar way, we can obtain (3.1b). This proves our assertions. $\square$

Algorithm 3.1. For arbitrarily chosen initial points $x_{0}, y_{0} \in K_{r}$, compute the sequences $\left\{x_{n}\right\}$ and $\left\{y_{n}\right\}$ in the following way:

$$
\begin{aligned}
& x_{n+1}=\left(1-\alpha_{n}\right) x_{n}+\alpha_{n} P_{K_{r}}\left[g\left(y_{n}\right)-\rho T_{1}\left(y_{n}, x_{n}\right)\right], \quad \rho>0, \\
& y_{n+1}=\left(1-\beta_{n}\right) x_{n+1}+\beta_{n} P_{K_{r}}\left[h\left(x_{n+1}\right)-\eta T_{2}\left(x_{n+1}, y_{n}\right)\right], \quad \eta>0,
\end{aligned}
$$

where $\left\{\alpha_{n}\right\},\left\{\beta_{n}\right\}$ are two sequences in $[0,1]$.

If $\beta_{n}=1, K_{r}=K$, then Algorithm 3.1 reduces to the following explicit projection method for solving the system of variational inequalities (2.3), which is mainly due to Noor and Noor [14]:

Algorithm 3.2. For arbitrarily chosen initial points $x_{0}, y_{0} \in K$, compute the sequences $\left\{x_{n}\right\}$ and $\left\{y_{n}\right\}$ in the following way:

$$
\begin{aligned}
& x_{n+1}=\left(1-\alpha_{n}\right) x_{n}+\alpha_{n} P_{K}\left[g\left(y_{n}\right)-\rho T_{1}\left(y_{n}, x_{n}\right)\right], \quad \rho>0, \\
& y_{n+1}=P_{K}\left[h\left(x_{n+1}\right)-\eta T_{2}\left(x_{n+1}, y_{n}\right)\right], \quad \eta>0,
\end{aligned}
$$

where $\left\{\alpha_{n}\right\},\left\{\beta_{n}\right\}$ are two sequences in $[0,1]$.

If $g=h=I, K_{r}=K$, and $T_{1}, T_{2}: K \rightarrow K$ are two univariate nonlinear operators, then Algorithm 3.1 reduces to the following explicit projection method for solving the system of variational inequalities (2.4):

Algorithm 3.3. For arbitrarily chosen initial points $x_{0}, y_{0} \in K$, compute the sequences $\left\{x_{n}\right\}$ and $\left\{y_{n}\right\}$ in the following way:

$$
\begin{aligned}
& x_{n+1}=\left(1-\alpha_{n}\right) x_{n}+\alpha_{n} P_{K}\left[y_{n}-\rho T_{1}\left(y_{n}\right)\right], \quad \rho>0, \\
& y_{n+1}=\left(1-\beta_{n}\right) x_{n+1}+\beta_{n} P_{K}\left[x_{n+1}-\eta T_{2}\left(x_{n+1}\right)\right], \quad \eta>0,
\end{aligned}
$$

where $\left\{\alpha_{n}\right\},\left\{\beta_{n}\right\}$ are two sequences in [0,1]. Algorithm 3.3 extends and improves the two-step projection methods of Verma [13].

If $g=h, T_{1}=T_{2}=T$ is the univariate nonlinear operator, we again use the fixed point formulation (3.1) to suggest and analyze the following explicit projection method, known as Mann iteration:

Algorithm 3.4. For arbitrarily chosen initial points $x_{0} \in K_{r}$, compute the sequence $\left\{x_{n}\right\}$ in the following way:

$$
x_{n+1}=\left(1-\alpha_{n}\right) x_{n}+\alpha_{n} P_{K_{r}}\left[g\left(x_{n}\right)-\rho T x_{n}\right], \quad \rho>0,
$$

where $\left\{\alpha_{n}\right\}$ is a sequence in $[0,1]$.

Remark 3.1. Algorithm 3.4 includes the projection methods of Noor [10] as special cases.

\section{Main results}

We now consider the convergence analysis of Algorithm 3.1, and this is the main motivation of our next result. In a similar way, we consider the convergence criteria of other algorithms. 
Theorem 4.1. Let $P_{K_{r}}$ be a Lipschitz continuous operator with constant $\delta=\frac{r}{r-r^{\prime}}$. Let $T_{i}: K_{r} \times K_{r} \rightarrow K_{r}$ be $r_{i}$-strongly monotone and $\mu_{i}$-Lipschitz continuous in the first variable, $i=1,2$, and $g, h: K_{r} \rightarrow K_{r}$ be strongly monotone with constants $r_{3}, r_{4}$ and Lipschitz continuous with constants $\mu_{3}, \mu_{4}$, respectively. If there exist constants $\rho, \eta>$ 0 such that

$$
\begin{aligned}
& \left|\rho-\frac{r_{1}}{\mu_{1}^{2}}\right|<\frac{\sqrt{\delta^{2} r_{1}^{2}-\mu_{1}^{2}\left[\delta^{2}-\left(1-\delta k_{1}\right)^{2}\right]}}{\delta \mu_{1}^{2}}, \quad \delta r_{1}>\mu_{1} \sqrt{\delta^{2}-\left(1-\delta k_{1}\right)^{2}}, \delta k_{1}<1, \\
& \left|\eta-\frac{r_{2}}{\mu_{2}^{2}}\right|<\frac{\sqrt{\delta^{2} r_{2}^{2}-\mu_{2}^{2}\left[\delta^{2}-\left(1-\delta k_{2}\right)^{2}\right]}}{\delta \mu_{2}^{2}}, \quad \delta r_{2}>\mu_{2} \sqrt{\delta^{2}-\left(1-\delta k_{2}\right)^{2}}, \delta k_{2}<1,
\end{aligned}
$$

where

$$
k_{1}=\sqrt{1-2 r_{3}+\mu_{3}^{2}}, \quad k_{2}=\sqrt{1-2 r_{4}+\mu_{4}^{2}}
$$

and $\alpha_{n}, \beta_{n} \in[0,1], \sum_{n=0}^{\infty} \alpha_{n}=\infty, \sum_{n=0}^{\infty}\left(1-\beta_{n}\right)<\infty$, then the sequences $\left\{x_{n}\right\}$ and $\left\{y_{n}\right\}$ obtained from Algorithm 3.1 converges to a solution of the system of general nonconvex variational inequalities (2.1), respectively.

Proof. Let $x^{*}, y^{*} \in K_{r}$ be a solution of (2.1). From (3.1a) and (3.3a) and the Lipschitz continuous property of operator $P_{K_{r}}$, we can obtain

$$
\begin{aligned}
\left\|x_{n+1}-x^{*}\right\|= & \left\|\left(1-\alpha_{n}\right)\left(x_{n}-x^{*}\right)+\alpha_{n}\left\{P_{K_{r}}\left[g\left(y_{n}\right)-\rho T_{1}\left(y_{n}, x_{n}\right)\right]-P_{K_{r}}\left[g\left(y^{*}\right)-\rho T_{1}\left(\gamma^{*}, x^{*}\right)\right]\right\}\right\| \\
\leq & \left(1-\alpha_{n}\right)\left\|x_{n}-x^{*}\right\|+\alpha_{n}\left\|P_{K_{r}}\left[g\left(y_{n}\right)-\rho T_{1}\left(y_{n}, x_{n}\right)\right]-P_{K_{r}}\left[g\left(y^{*}\right)-\rho T_{1}\left(y^{*}, x^{*}\right)\right]\right\| \\
\leq & \left(1-\alpha_{n}\right)\left\|x_{n}-x^{*}\right\|+\alpha_{n} \delta\left\|g\left(y_{n}\right)-g\left(\gamma^{*}\right)-\rho\left[T_{1}\left(y_{n}, x_{n}\right)-T_{1}\left(y^{*}, x^{*}\right)\right]\right\| \\
\leq & \left(1-\alpha_{n}\right)\left\|x_{n}-x^{*}\right\|+\alpha_{n} \delta\left\|g\left(y_{n}\right)-g\left(\gamma^{*}\right)-\left(y_{n}-\gamma^{*}\right)\right\|+\alpha_{n} \delta \|\left(y_{n}-y^{*}\right)- \\
& \rho\left[T_{1}\left(y_{n}, x_{n}\right)-T_{1}\left(\gamma^{*}, x^{*}\right)\right] \| .
\end{aligned}
$$

Since the operator $T_{1}$ is $r_{1}$-strongly monotone and $\mu_{1}$-Lipschitz continuous definition in the first variable, it follows that

$$
\begin{aligned}
& \left\|y_{n}-y^{*}-\rho\left[T_{1}\left(y_{n}, x_{n}\right)-T_{1}\left(\gamma^{*}, x^{*}\right)\right]\right\|^{2} \\
& \quad=\left\|y_{n}-\gamma^{*}\right\|^{2}-2 \rho\left\langle T_{1}\left(y_{n}, x_{n}\right)-T_{1}\left(\gamma^{*}, x^{*}\right), y_{n}-y^{*}\right\rangle+\rho^{2}\left\|T_{1}\left(y_{n}, x_{n}\right)-T_{1}\left(y^{*}, x^{*}\right)\right\|^{2} \\
& \quad \leq\left\|y_{n}-\gamma^{*}\right\|^{2}-2 \rho r_{1}\left\|y_{n}-\gamma^{*}\right\|^{2}+\rho^{2}\left\|T_{1}\left(y_{n}, x_{n}\right)-T_{1}\left(\gamma^{*}, x^{*}\right)\right\|^{2} \\
& \quad \leq\left(1-2 \rho r_{1}+\rho^{2} \mu_{1}^{2}\right)\left\|y_{n}-\gamma^{*}\right\|^{2}
\end{aligned}
$$

which implies that

$$
\left\|y_{n}-\gamma^{*}-\rho\left[T_{1}\left(y_{n}, x_{n}\right)-T_{1}\left(\gamma^{*}, x^{*}\right)\right]\right\| \leq \sqrt{1-2 \rho r_{1}+\rho^{2} \mu_{1}^{2}}\left\|y_{n}-\gamma^{*}\right\| .
$$

In a similar way, we have (note that $\mu_{3} \geq r_{3}$, from Remark 2.3)

$$
\left\|g\left(y_{n}\right)-g\left(\gamma^{*}\right)-\left(y_{n}-y^{*}\right)\right\| \leq \sqrt{1-2 r_{3}+\mu_{3}^{2}}\left\|y_{n}-\gamma^{*}\right\| .
$$

Consequently, from (4.3), (4.5), and (4.6), we have

$$
\begin{aligned}
\left\|x_{n+1}-x^{*}\right\| & \leq\left(1-\alpha_{n}\right)\left\|x_{n}-x^{*}\right\|+\alpha_{n} \delta\left(k_{1}+\sqrt{1-2 \rho r_{1}+\rho^{2} \mu_{1}^{2}}\right)\left\|y_{n}-\gamma^{*}\right\| \\
& =\left(1-\alpha_{n}\right)\left\|x_{n}-x^{*}\right\|+\alpha_{n} \theta_{1}\left\|y_{n}-\gamma^{*}\right\|
\end{aligned}
$$


where $\theta_{1}=\delta\left(k_{1}+\sqrt{1-2 \rho r_{1}+\rho^{2} \mu_{1}^{2}}\right), k_{1}=\sqrt{1-2 r_{3}+\mu_{3}^{2}}$. From (4.1), we obtain that $\theta_{1} \in(0,1)$.

On the other hand, it follows from (3.1b) and (3.3b) that

$$
\begin{aligned}
\left\|y_{n+1}-y^{*}\right\| \leq & \left(1-\beta_{n}\right)\left\|x_{n+1}-\gamma^{*}\right\|+\beta_{n}\left\|P_{K_{r}}\left[h\left(x_{n+1}\right)-\eta T_{2}\left(x_{n+1}, y_{n}\right)\right]-P_{K_{r}}\left[h\left(x^{*}\right)-\eta T_{2}\left(x^{*}, y^{*}\right)\right]\right\| \\
\leq & \left(1-\beta_{n}\right)\left\|x_{n+1}-\gamma^{*}\right\|+\beta_{n} \delta\left\|h\left(x_{n+1}\right)-h\left(x^{*}\right)-\eta\left[T_{2}\left(x_{n+1}, y_{n}\right)-T_{2}\left(x^{*}, y^{*}\right)\right]\right\| \\
\leq & \left(1-\beta_{n}\right)\left\|x_{n+1}-x^{*}\right\|+\left(1-\beta_{n}\right)\left\|x^{*}-\gamma^{*}\right\|+\beta_{n} \delta\left\|h\left(x_{n+1}\right)-h\left(x^{*}\right)-\left(x_{n+1}-x^{*}\right)\right\|+ \\
& \beta_{n} \delta\left\|x_{n+1}-x^{*}-\eta\left[T_{2}\left(x_{n+1}, y_{n}\right)-T_{2}\left(x^{*}, y^{*}\right)\right]\right\| .
\end{aligned}
$$

Similarly, from the properties defined on $T_{2}$ in the first variable, we have

$$
\begin{aligned}
& \left\|x_{n+1}-x^{*}-\eta\left[T_{2}\left(x_{n+1}, y_{n}\right)-T_{2}\left(x^{*}, y^{*}\right)\right]\right\|^{2} \\
& \quad=\left\|x_{n+1}-x^{*}\right\|^{2}-2 \eta\left\langle T_{2}\left(x_{n+1}, y_{n}\right)-T_{2}\left(x^{*}, y^{*}\right), x_{n+1}-x^{*}\right\rangle+\eta^{2}\left\|T_{2}\left(x_{n+1}, y_{n}\right)-T_{2}\left(x^{*}, y^{*}\right)\right\|^{2} \\
& \quad \leq\left\|x_{n+1}-x^{*}\right\|^{2}-2 \eta r_{2}\left\|x_{n+1}-x^{*}\right\|^{2}+\eta^{2}\left\|T_{2}\left(x_{n+1}, y_{n}\right)-T_{2}\left(x^{*}, y^{*}\right)\right\|^{2} \\
& \quad \leq\left(1-2 \eta r_{2}+\eta^{2} \mu_{2}^{2}\right)\left\|x_{n+1}-x^{*}\right\|^{2}
\end{aligned}
$$

and

$$
\left\|h\left(x_{n+1}\right)-h\left(x^{*}\right)-\left(x_{n+1}-x^{*}\right)\right\|^{2} \leq\left(1-2 r_{4}+\mu_{4}^{2}\right)\left\|x_{n+1}-x^{*}\right\|^{2}
$$

Moreover, from (4.8)-(4.10), we have

$$
\begin{aligned}
\left\|y_{n+1}-\gamma^{*}\right\| & \leq\left(1-\beta_{n}\right)\left\|x_{n+1}-x^{*}\right\|+\left(1-\beta_{n}\right)\left\|x^{*}-y^{*}\right\|+\beta_{n} \delta\left(k_{2}+\sqrt{1-2 \eta r_{2}+\eta^{2} \mu_{2}^{2}}\right)\left\|x_{n+1}-x^{*}\right\| \\
& \leq\left(1-\beta_{n}\right)\left\|x_{n+1}-x^{*}\right\|+\left(1-\beta_{n}\right)\left\|x^{*}-y^{*}\right\|+\beta_{n} \theta_{2}\left\|x_{n+1}-x^{*}\right\|
\end{aligned}
$$

where $\theta_{2}=\delta\left(k_{2}+\sqrt{1-2 \eta r_{2}+\eta^{2} \mu_{2}^{2}}\right), k_{2}=\sqrt{1-2 r_{4}+\mu_{4}^{2}}$. From (4.2), we obtain that $\theta_{2} \in(0,1)$. For all $n \geq 1$, it follows from (4.11) that

$$
\begin{aligned}
\left\|y_{n}-\gamma^{*}\right\| & \leq\left(1-\beta_{n-1}\right)\left\|x_{n}-x^{*}\right\|+\left(1-\beta_{n-1}\right)\left\|x^{*}-\gamma^{*}\right\|+\beta_{n-1} \theta_{2}\left\|x_{n}-x^{*}\right\| \\
& =\left[1-\left(1-\theta_{2}\right) \beta_{n-1}\right]\left\|x_{n}-x^{*}\right\|+\left(1-\beta_{n-1}\right)\left\|x^{*}-y^{*}\right\| .
\end{aligned}
$$

Substituting (4.12) into (4.7), we have (note that $\theta_{1}, \theta_{2} \in(0,1)$ )

$$
\begin{aligned}
\left\|x_{n+1}-x^{*}\right\| & \leq\left(1-\alpha_{n}\right)\left\|x_{n}-x^{*}\right\|+\alpha_{n} \theta_{1}\left\{\left[1-\left(1-\theta_{2}\right) \beta_{n-1}\right]\left\|x_{n}-x^{*}\right\|+\left(1-\beta_{n-1}\right)\left\|x^{*}-y^{*}\right\|\right\} \\
& \leq\left(1-\alpha_{n}\right)\left\|x_{n}-x^{*}\right\|+\alpha_{n} \theta_{1}\left\|x_{n}-x^{*}\right\|+\alpha_{n}\left(1-\beta_{n-1}\right) \theta_{1}\left\|x^{*}-\gamma^{*}\right\| \\
& \leq\left[1-\left(1-\theta_{1}\right) \alpha_{n}\right]\left\|x_{n}-x^{*}\right\|+\alpha_{n}\left(1-\beta_{n-1}\right)\left\|x^{*}-y^{*}\right\| .
\end{aligned}
$$

Since $1-\theta_{1}>0, \sum_{n=0}^{\infty} \alpha_{n}=\infty$ and $\sum_{n=0}^{\infty}\left(1-\beta_{n}\right)<\infty$, we apply Lemma 2.3 to get

$$
\lim _{n \rightarrow \infty}\left\|x_{n}-x^{*}\right\|=0
$$

Combining (4.12) and (4.14), we have

$$
\lim _{n \rightarrow \infty}\left\|y_{n}-y^{*}\right\|=0
$$

It follows that $\lim _{n \rightarrow \infty} x_{n}=x^{*}, \lim _{n \rightarrow \infty} y_{n}=y^{*}$, satisfying the general system of nonconvex variational inequalities (2.1). This completes the proof. $\square$

Theorem 4.2. Let $K$ be a nonempty and convex subset of Hilbert space $H$. Let $T_{i}: K$ $\times K \rightarrow K$ be $r_{i}$-strongly monotone and $\mu_{i}$-Lipschitz continuous in the first variable, $i=$ 1,2 , and $g, h: K \rightarrow K$ be strongly monotone with constants $r_{3}, r_{4}$ and Lipschitz continuous with constants $\mu_{3}, \mu_{4}$, respectively. If there exist constants $\rho, \eta>0$ such that 


$$
\begin{aligned}
& \left|\rho-\frac{r_{1}}{\mu_{1}^{2}}\right|<\frac{\sqrt{r_{1}^{2}-\mu_{1}^{2}\left(2 k_{1}-k_{1}^{2}\right)}}{\mu_{1}^{2}}, \quad r_{1}>\mu_{1} \sqrt{2 k_{1}-k_{1}^{2}}, k_{1}<1, \\
& \left|\eta-\frac{r_{2}}{\mu_{2}^{2}}\right|<\frac{\sqrt{r_{2}^{2}-\mu_{2}^{2}\left(2 k_{2}-k_{2}^{2}\right)}}{\mu_{2}^{2}}, \quad r_{2}>\mu_{2} \sqrt{2 k_{2}-k_{2}^{2}}, k_{2}<1,
\end{aligned}
$$

where

$$
k_{1}=\sqrt{1-2 r_{3}+\mu_{3}^{2}}, \quad k_{2}=\sqrt{1-2 r_{4}+\mu_{4}^{2}}
$$

and $\alpha_{n} \in[0,1], \sum_{n=0}^{\infty} \alpha_{n}=\infty$, then the sequences $\left\{x_{n}\right\}$ and $\left\{y_{n}\right\}$ obtained from Algorithm 3.2 converges to a solution of the system of general variational inequalities (2.3), respectively.

Proof. If $K_{r}=K, \beta_{n}=1$, Algorithm 3.1 reduces to Algorithm 3.2. Moreover, we can obtain $r=\infty$ and $\delta=1$ from Remark 2.1, and $\sum_{n=0}^{\infty}\left(1-\beta_{n}\right)=0$. Then the conclusion follows immediately from Theorem 4.1. This completes the proof. $\square$

Theorem 4.3. Let $K$ be a nonempty and convex subset of Hilbert space $H$, and $T_{i}: K$ $\rightarrow K$ be $r_{i}$-strongly monotone and $\mu_{i}$-Lipschitz continuous, $i=1,2$. If there exist constants $\rho, \eta$ such that

$$
0<\rho<\frac{2 r_{1}}{\mu_{1}^{2}}, \quad 0<\eta<\frac{2 r_{2}}{\mu_{2}^{2}} .
$$

and $\alpha_{n}, \beta_{n} \in[0,1], \sum_{n=0}^{\infty} \alpha_{n}=\infty, \sum_{n=0}^{\infty}\left(1-\beta_{n}\right)<\infty$, then the sequences $\left\{x_{n}\right\}$ and $\left\{y_{n}\right\}$ obtained from Algorithm 3.3 converges to a solution of the system of variational inequalities (2.4), respectively.

Proof. If $g=h=I, K_{r}=K$, Algorithm 3.1 reduces to Algorithm 3.3. Moreover, we can obtain $r=\infty$ and $\delta=1$ from Remark 2.1, and $k_{1}=k_{2}=0$ from Remark $2.2\left(r_{3}=\right.$ $\left.\mu_{3}=r_{4}=\mu_{4}=1\right)$. Then the conclusion follows immediately from Theorem 4.1. This completes the proof. $\square$

\section{Acknowledgements}

This study was supported by the National Science Foundation of China (11001287), Natural Science Foundation Project of Chongqing (CSTC 2010BB9254) and Science and Technology Research Project of Chongqing Municipal Education Commission (KJ 110701).

\footnotetext{
Author details

${ }^{1}$ College of Mathematics and Statistics, Chongqing Technology and Business University, Chongqing 400067, China ${ }^{2}$ College of Computer Science and Information Engineering, Chongqing Technology and Business University, Chongqing 400067, China
}

\section{Authors' contributions}

DJW carried out the primary studies for the new system of general nonconvex variational inequalities, participated in the design of projection methods and drafted the manuscript. XJL participated in the design of the study and performed the nonconvex analysis. QFG participated in the convergence analysis and coordination. All authors read and approved the final manuscript.

\section{Competing interests}

The authors declare that they have no competing interests.

Received: 23 July 2011 Accepted: 13 April 2012 Published: 13 April 2012

\section{References}

1. Stampacchia, G: Formes bilinearies coercivities sur les ensembles convexes. C R Acad Sci Paris. 258, 4413-4416 (1964) 
2. Noor, MA: Some developments in general variational inequalities. Appl Math Comput. 152, 199-277 (2004). doi:10.1016/ S0096-3003(03)00558-7

3. Bounkhel, M, Tadj, L, Hamdi, A: Iterative schemes to solve nonconvex variational problems. J Inequal Pure Appl Math. 4, 1-14 (2003)

4. Noor, MA: On implicit methods for nonconvex variational inequalities. J Optim Theory Appl. 147, 411-417 (2010). doi:10.1007/s10957-010-9717-y

5. Pang, LP, Shen, J, Song, HS: A modified predictor-corrector algorithm for solving non-convex generalized variational inequalities. Comput Math Appl. 54, 319-325 (2007). doi:10.1016/j.camwa.2006.07.010

6. Verma, RU: Generalized system for relaxed cocoercive variational inequalities and projection methods. J Optim Theory Appl. 121(1), 203-210 (2004)

7. Noor, MA: Some iterative methods for nonconvex variational inequalities. Comput Math Model. 21(1), 97-108 (2010). doi:10.1007/s10598-010-9057-7

8. Clarke, FH, Ledyaev, YS, Wolenski, PR: Nonsmooth Analysis and Control Theory. Springer, Berlin (1998)

9. Poliquin, RA, Rockafellar, RT, Thibault, L: Local differentiability of distance functions. Trans Am Math Soc. 352, 5231-5249 (2000). doi:10.1090/S0002-9947-00-02550-2

10. Noor, MA: Projection methods for nonconvex variational inequalities. Optim Lett. 3, 411-418 (2009). doi:10.1007/s11590009-0121-1

11. Noor, MA: Iterative methods for general nonconvex variational inequalities. Albanian J Math. 3(1), 117-127 (2009)

12. Huang, Z, Noor, MA: An explicit projection method for a system of nonlinear variational inequalities with different $(\gamma, r)$ cocoercive mappings. Appl Math Comput. 190, 356-361 (2007). doi:10.1016/j.amc.2007.01.032

13. Verma, RU: General Convergence analysis for two-step projection methods and applications to variational problems Appl Math Lett. 18, 1286-1292 (2005). doi:10.1016/j.aml.2005.02.026

14. Noor, MA, Noor, Kl: Projection algorithms for solving system of general variational inequalities. Nonlinear Anal. 70 , 2700-2706 (2009). doi:10.1016/j.na.2008.03.057

15. Wen, DJ: Projection methods for a generalized system of nonconvex variational inequalities with different nonlinear operators. Nonlinear Anal. 73, 2292-2297 (2010). doi:10.1016/j.na.2010.06.010

16. Ceng, LC, Teboulle, M, Yao, JC: Weak convergence of an iterative method for pseudomonotone variational inequalities and fixed-point problems. J Optim Theory Appl. 146, 19-31 (2010). doi:10.1007/s10957-010-9650-0

17. Wen, DJ: Strong convergence theorems for equilibrium problems and $k$-strict pseudo-contractions in Hilbert spaces. Abstract Appl Anal 2011 (2011). Article ID 276874, doi:10.1155/2011/276874

18. Xu, HK: Iterative algorithms for nonlinear operators. J Lond Math Soc. 2, 240-256 (2002)

doi:10.1186/1687-1812-2012-59

Cite this article as: Wen et al: Convergence analysis of projection methods for a new system of general

nonconvex variational inequalities. Fixed Point Theory and Applications 2012 2012:59.

\section{Submit your manuscript to a SpringerOpen ${ }^{\circ}$ journal and benefit from:}

- Convenient online submission

- Rigorous peer review

- Immediate publication on acceptance

- Open access: articles freely available online

- High visibility within the field

- Retaining the copyright to your article

Submit your next manuscript at $\gg$ springeropen.com 